\title{
Instability of the Boundary in the Billiard Ball Problem
}

\author{
Andrea Hubacher
}

ETH Zürich, CH-8092 Zürich, Switzerland

\begin{abstract}
We consider the billiard ball problem in the interior of a plane closed convex $C^{1}$ curve which is piecewise $C^{2}$. If the curvature has a discontinuity, then the boundary is unstable, i.e. no caustics exist near the boundary. However, in the interior there can exist caustics, as we show by an example.
\end{abstract}

We consider the billiard ball problem in the interior of a plane closed convex $C^{1}$ curve. We assume that the second derivatives of the curve exist and are continuous except in finitely many points, where the limits of the second derivatives exist from both sides, but where the curvature is discontinuous. Furthermore we assume the curvature to be strictly positive and uniformly bounded. This situation is illustrated by a convex boundary consisting of circular arcs matching with their tangents (see [4]).

Denote the boundary curve of the billiard table by $C$ and its total length by $L$. The billiard ball problem can be described in the two coordinates arc length $s$ and angle $t$ between outgoing billiard ray and positive tangent direction in a point of reflection at the boundary. Associating to such a pair of coordinates $(s, t)$ the corresponding pair $\left(s_{1}, t_{1}\right)$ of the next reflection point (see Fig. 1) gives rise to a homeomorphism from the annulus $(\mathbb{R} / L \mathbb{Z}) \times[0, \pi]$ to itself, which we will call the billiard map (see [1]).

Fig. 1. The billiard map

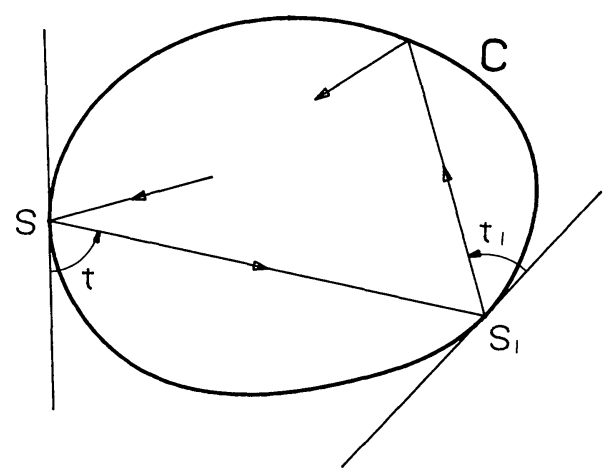


It is easy to see that the billiard map is orientation preserving and maps each boundary component of the annulus $(\mathbb{R} / L \mathbb{Z}) \times[0, \pi]$ into itself. As is well known, it furthermore leaves the measure $\sin (s) d s d t$ invariant and satisfies the so-called twist condition: $s_{1}$ depends strictly monotonously on $t$. For the class of twist maps which leave some regular measure invariant (regular means here: the measure of any nonempty open set is strictly positive), the following theorem of G. D. Birkhoff holds (see Herman [5, appendix of Fathi to Chap. I] for a detailed proof):

Birkhoff's Theorem. Let $H$ be an orientation preserving and boundary component preserving twist homeomorphism from the annulus $A=T^{1} \times[0,1]$ into itself which preserves a regular measure. Assume $U$ to be an open subset of A homeomorphic to $T^{1} \times\left[0,1\left[\right.\right.$ and such that $\partial U \cap T^{1} \times\{1\}=\Phi$, where $\partial U$ is the boundary of $U$ with respect to $A$. If $U$ is invariant under $H$ (i.e. $H(U)=U$ ), then $\partial U$ is the graph of a continuous function from $T^{1}$ into $] 0,1[. \partial U$ is even a Lipschitz curve, provided the twist of $H$ is uniform.

Therefore, looking for not zero homotopic subsets of the annulus which are invariant under the billiard map is equivalent to looking for invariant curves of the form $t=t(s)$; absence of invariant curves precludes the existence of any nontrivial stable domain not homotopic to zero.

A convex curve lying in the interior of the billiard table with the property that any billiard trajectory tangential to it stays tangential after reflection at the boundary, will be called a caustic. Existence of caustics gives rise to existence of invariant curves for the billiard map.

Theorem. Suppose that the boundary $C$ of the billiard table has at least one discontinuity in the curvature as described above. Then the boundary circle $\mathbb{R} / L \mathbb{Z} \times\{0\}$ of the annulus $(\mathbb{R} / L \mathbb{Z}) \times[0, \pi]$ is an isolated invariant curve of the billiard map; hence caustics cannot accumulate at the boundary curve $C$.

For our result it is essential that the edge $C$ of the billiard table is not smooth: A theorem of Lazutkin [6] states that a boundary which is sufficiently smooth and has strictly positive curvature possesses in any neighborhood a Cantor set of caustics. (Lazutkin assumed in his original work very high differentiability conditions on the boundary; e.g. Douady [2] lowered them to $C^{6}$, he conjectures that $C^{4}$ is sufficient.)

Halpern [3] constructed an example which is globally $C^{2}$ but not $C^{3}$ possessing an orbit approaching the boundary asymptotically within a finite amount of time. He also proves that billiards with a $C^{3}$ boundary do not possess such orbits.

Very interesting related work has been done by Mather $[8,9]$ who proved that any Birkhoff region of instability of an area preserving monotone twist map possesses certain chaotic properties similar to the shadowing lemma for hyperbolic systems. From this work it follows that any billiard with unstable boundary possesses an orbit approaching the boundary asymptotically; in particular, if there is not any caustic in the interior of the billiard table, then there exists an orbit asymptotic to the boundary in opposite directions as the number of forward or backward reflections at the boundary goes to infinity. From Halpern's work it follows that this orbit will need infinite time to approach the boundary, i.e. it will 

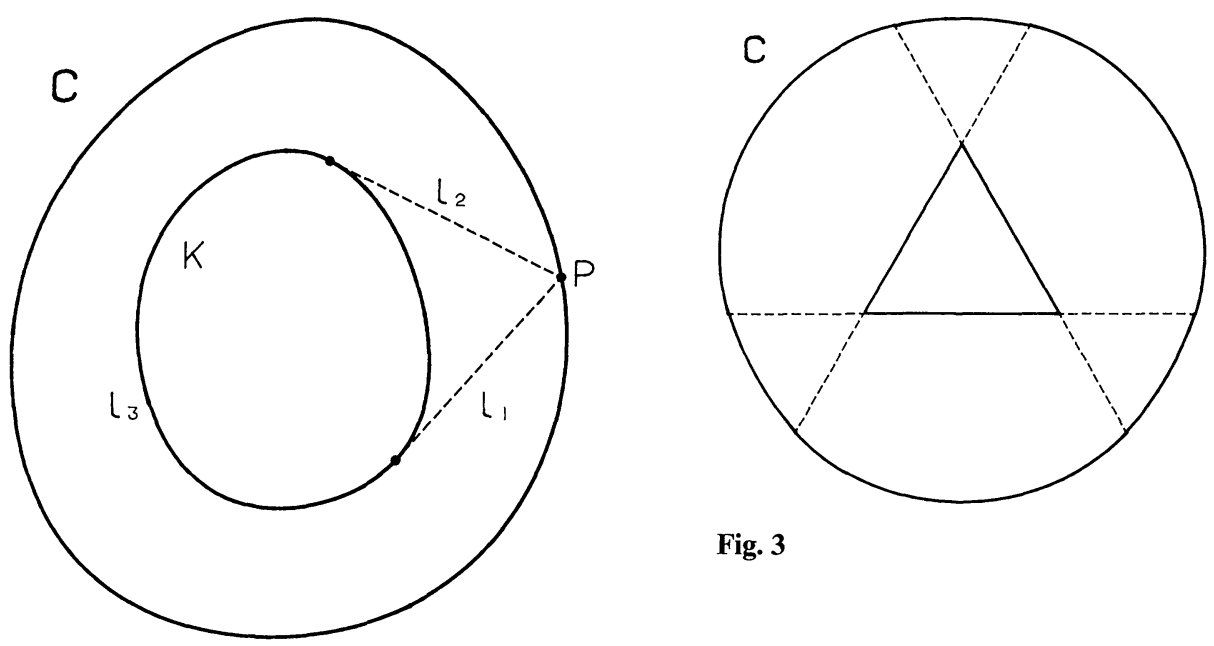

Fig. 3

Fig. 2. Wire construction of a billiard table $C$ for a given caustic $K$. $C=$ the set of all $P$ for which $l_{1}+l_{2}+l_{3}=l$, where $l>$ length of $K$ is a given constant

infinitely often go around the whole annulus, if the boundary is $C^{3}$ or piecewise $C^{3}$ with third derivatives uniformly bounded.

Mather [7] also showed that a zero of the curvature implies global nonexistence of invariant curves; we therefore assume the curvature to be uniformly bounded away from zero.

Our result applies to the Benettin-Strelcyn oval billiard (see Hénon and Wisdom [4]).

A discontinuity of the curvature implies only nonexistence of invariant curves at the boundary, not in the interior of the annulus. This is demonstrated by the following example, which we owe to Jürgen Moser: Given a convex curve $K$, one can obtain any billiard table possessing this curve $K$ as a caustic by a construction analogous to the string construction of the ellipse (see Fig. 2). This construction smoothens, i.e. even if the curve $K$ is only piecewise $C^{1}$ the so constructed curve $C$ always will be globally $C^{1}$. This can easily be seen geometrically (observe that in Fig. 2 the angles between the two tangents at $K$ and the curve $C$ are equal). We take an equilateral triangle, with side length $a$, and construct the billiard table $C$ with a string of length $l>3 a$ as described above. The resulting curve $C$, consisting of six pieces of ellipses (see Fig. 3), is globally $C^{1}$, but the curvature is discontinuous in the six points where the ellipses are glued together. The billiard problem in the interior of $C$ possesses at least one invariant curve in the interior, since the triangle is a (degenerate) caustic. $C$ can be arbitrarily close to a circle by making $a / l$ small.

\section{Proof of the Theorem}

1st Step. First we prove that any point of discontinuity of the curvature possesses a neighborhood through which there pass no nontrivial invariant curves.

Assume $s=0$ to be a point where the curvature jumps. Denote the curvature by $k(s)$. We may assume $k(0-)>k(0+)$. Let $r=k(0-)^{-1}$ and $R=k(0+)^{-1}$. Since $k$ is uniformly bounded away from zero, both $r$ and $R$ are finite. 
Fig. 4

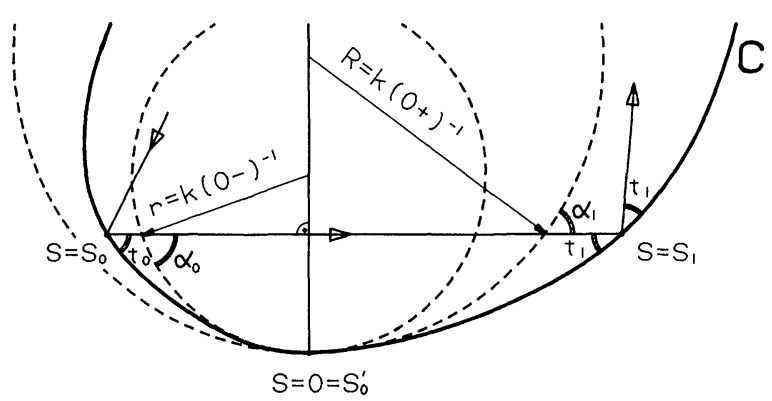

The billiard map can be lifted to a homeomorphism $\phi: \mathbb{R} \times[0, \pi] \rightarrow \mathbb{R} \times[0, \pi]$, $(s, t) \rightarrow\left(s_{1}, t_{1}\right)=\phi(s, t)$ which is uniquely determined by requiring $\phi(s, 0)=(s, 0)$ for all $s \in \mathbb{R}$.

In order to study a neighborhood of the singular boundary point it is sufficient to study a neighborhood of $(0,0)$ in $\mathbb{R} \times[0, \pi]$. Proceeding indirectly, we assume that through any neighborhood of the point $(0,0)$ there passes at least one nontrivial $\Phi$-invariant curve. Let $V$ be a neighborhood of $(0,0)$ in $\mathbb{R} \times[0, \pi]$ such that both the osculating circles in the point $s=0$ of $C$ approximate $C$ for $s \in V \cap \mathbb{R} \times\{0\}$ up to $o(s)$ as $s \rightarrow 0$. Denote the nontrivial invariant curve passing through $V$ by $\Gamma$. Since $\Gamma$ is continuous, it contains a point $Q=\left(s_{0}, t_{0}\right)$ such that the billiard trajectory from $Q$ to $\phi Q=\left(s_{1}, t_{1}\right)$ cuts the inner normal of $C$ in the point $s=0$ perpendicularly (see Fig. 4). The billiard ray from $Q$ to $\phi Q$ cuts the osculating circle of radius $r$ by an angle $\alpha_{0}$ and the other circle of radius $R$ by an angle $\alpha_{1}$. Evidently (see Fig. 4!)

$$
R \cos \alpha_{1}=R-r+r \cos \alpha_{0} .
$$

Define $f(\alpha):=\arccos (1-r / R+r / R \cos \alpha)$; then we have for $\alpha>0$

$$
f^{\prime}(\alpha)=\sqrt{r / R} / \sqrt{1+(1-r / R) \tan ^{2}(\alpha / 2)}<\sqrt{r / R},
$$

and therefore

$$
\alpha_{1}=f\left(\alpha_{0}\right)=\alpha_{0} \int_{0}^{1} f^{\prime}\left(\tau \alpha_{0}\right) d \tau<\alpha_{0} \sqrt{r / R} .
$$

By approximation properties of the osculating circle, $\alpha_{0}=t_{0}+o\left(t_{0}\right)$ and $t_{1}=\alpha_{1}$ $+o\left(\alpha_{1}\right)$ as the angles tend to zero; so $(*)$ implies that $t_{1}<\sqrt{r / R} t_{0}+o\left(t_{0}\right)$, from which we conclude that for $V$ small enough $t_{1}<t_{0}$ and

$$
t_{0}-t_{1}>\frac{1}{2}(1-\sqrt{r / R}) t_{0}>\frac{1}{2}(1-\sqrt{r / R}) t_{1} .
$$

Now we consider all possible orbits through $s_{0}^{\prime}=0$. These orbits represent the billiard trajectories reflecting at the boundary $C$ exactly in the point where the curvature is discontinuous. Define for $\left(s_{0}, t_{0}\right)$, respectively $\left(s_{0}^{\prime}, t_{0}^{\prime}\right)$, the orbits $\left(s_{n}, t_{n}\right)$ $=\phi^{n}\left(s_{0}, t_{0}\right)$, respectively $\left(s_{n}^{\prime}, t_{n}^{\prime}\right)=\phi^{n}\left(s_{0}^{\prime}, t_{0}^{\prime}\right)$, for $n \in \mathbb{Z}$.

What we intend to show is, that for any choice of $t_{0}^{\prime}$ the graphs of the sequences $\left(s_{n}\right)_{n \in \mathbb{Z}}$ and $\left(s_{n}^{\prime}\right)_{n \in \mathbb{Z}}$ cross, i.e. $s_{0}<s_{0}^{\prime}$, but $s_{n}>s_{n}^{\prime}$ for some $n \neq 0$. Since Birkhoff's theorem forbids such a crossing, we will have reached a contradiction. 
To this end we will prove below that for any $\delta>0$ there exists a neighborhood $V$ of $(0,0)$, such that for $\left(\left(s_{n}, t_{n}\right)\right)_{n \in \mathbb{Z}}$ passing through $V$ the condition

$$
\left|t_{0}^{\prime}-t_{1}\right|<\delta t_{1}
$$

on $t_{0}^{\prime}$ is necessary to avoid a crossing of the orbits $\left(s_{n}\right)_{n \in \mathbb{Z}}$ and $\left(s_{n}^{\prime}\right)_{n \in \mathbb{Z}}$ for positive $n$, respectively

for negative $n$.

$$
\left|t_{0}^{\prime}-t_{0}\right|<\delta t_{0}
$$

Assume that (2) is proven. If the orbits $\left(s_{n}\right)_{n \in \mathbb{Z}}$ and $\left(s_{n}^{\prime}\right)_{n \in \mathbb{Z}}$ do not cross for positive $n,(2 \mathrm{a})$ is valid. With $\delta=\frac{1}{4}(1-\sqrt{r / R})$ we conclude from $t_{1}<t_{0}$ and (1) that $\left|t_{0}^{\prime}-t_{0}\right| \geqq t_{0}-t_{1}-\left|t_{0}^{\prime}-t_{1}\right|>\delta t_{0}$; hence (2b) is not satisfied, which means that the orbits cross for negative $n$. Analogously, if the orbits do not cross for negative $n$, they have to cross for positive $n$. So the sequences $\left(s_{n}\right)_{n \in \mathbb{Z}}$ and $\left(s_{n}^{\prime}\right)_{n \in \mathbb{Z}}$ cross, for any choice of $t_{0}^{\prime}$.

It remains to prove (2): The condition $s_{1}<s_{1}^{\prime}<s_{2}$ restricts $t_{0}^{\prime}$ to be small. Let $V_{R}=V \cap\{s>0\}$. Since $C$ is smooth near $s=0, \phi$ possesses on $V_{R}$ the asymptotic expansion

$$
\begin{aligned}
& s_{1}=s+2 k(s)^{-1} t+o(t) \\
& t_{1}=\quad t+o(t) \text { as } t \rightarrow 0 .
\end{aligned}
$$

Assume $0<\delta<1$ fixed. Set $m:=\inf _{V_{R}} 2 k(s)^{-1}$ and $M:=\sup _{V_{R}} 2 k(s)^{-1}$. Making $V$ smaller if necessary we can assume $m>(1-\delta) M$ (observe that $k$ is bounded away from zero).

Let $n$ be the smallest integer greater than $2 m /(m-(1-\delta) M)$. Since $n$ is fixed, all the iterates $\phi^{j}\left(s_{0}, t_{0}\right)$ and $\phi^{j}\left(s_{0}^{\prime}, t_{0}^{\prime}\right)$ stay in $V_{R}$ for $1 \leqq j \leqq n$ if the angles $t_{0}$ and $t_{0}^{\prime}$ are small enough.

In order to proceed indirectly, we assume that (2a) is not satisfied, i.e. either $t_{0}^{\prime}<(1-\delta) t_{1}$ or $t_{0}^{\prime}>(1+\delta) t_{1}$. In the case $t_{0}^{\prime}<(1-\delta) t_{1}$ we obtain by application of (3) and because $n$ is fixed that for small angles

$$
\begin{aligned}
& s_{n}>s_{n}-s_{1}=s_{1}+t_{1} \sum_{j=1}^{n-1} 2 k\left(s_{j}\right)^{-1}+o\left(t_{1}\right)>t_{1}(n-1) m+o\left(t_{1}\right), \\
& s_{n}^{\prime}=s_{n}^{\prime}-s_{0}^{\prime}=s_{0}^{\prime}+t_{0}^{\prime} \sum_{j=0}^{n-1} 2 k\left(s_{j}^{\prime}\right)^{-1}+o\left(t_{0}^{\prime}\right) \leqq t_{1}(1-\delta) n M+o\left(t_{1}\right),
\end{aligned}
$$

which implies for small angles, since $n>2 m /(m-(1-\delta) M)$, that $s_{n}>s_{n}^{\prime}$. This is a contradiction to the no crossing condition since $s_{0}<s_{0}^{\prime}$. In the case $t_{0}^{\prime}>(1+\delta) t_{1}$ we obtain analogously for small angles that $s_{n}^{\prime}>s_{n-1}$ which contradicts $s_{0}^{\prime}<s_{1}$. This proves (2a).

In order to prove (2b) replace $\phi$ on $V_{R}$ by $\phi^{-1}$ on $V_{L}=V \cap\{s<0\}$. This proves the first step.

2nd Step. We prove now the existence of a neighborhood of the whole boundary circle $\mathbb{R} / L \mathbb{Z} \times\{0\}$ through which there passes no invariant curve. To get a contradiction, we assume $(s, 0) \in \mathbb{R} / L \mathbb{Z} \times\{0\}$ to be an accumulation point of a sequence $\left(\Gamma_{n}\right)_{n \in \mathbb{N}}$ of nontrivial invariant curves. Since the curve $C$ is piecewise $C^{2}$ 
Fig. 5

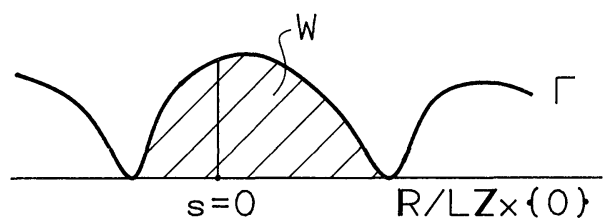

and $0<k<\infty$ uniformly, the twist is uniform. By Birkhoff's theorem the $\Gamma_{n}$ are Lipschitz, and possess therefore a subsequence converging to an invariant curve $\Gamma$ intersecting the boundary $\mathbb{R} / L \mathbb{Z} \times\{0\}$ of the annulus at least in the point $(s, 0)$. Since by the first step of the proof $\Gamma$ does not contain the singular point $(0,0)$, the two invariant curves $\Gamma$ and $\mathbb{R} / L \mathbb{Z} \times\{0\}$ enclose an open invariant set $W$ homeomorphic to the plane (see Fig. 5). Consider a vertical line in $W$, partitioning $W$ in two subsets of positive measure (see Fig. 5), and its image under the billiard map. Because of the twist property, we reach a contradiction to the measure preserving property of the billiard map.

Acknowledgement: It is a pleasure to thank Prof. J. P. Eckmann for his advice and for his constant encouragement.

\section{References}

1. Birkhoff, G.D.: Dynamical systems, Vol. IX. AMS Coll. 1927

2. Douady, R.: Thèse $3^{\text {ème }}$ cycle

3. Halpern, B.: Strange billiard tables. Trans. AMS 232, 297-305 (1977)

4. Hénon, M., Wisdom, J.: The Benettin-Strelcyn oval billiard revisited. Physica 8D, 157-169 (1983)

5. Herman, M.R.: Sur les courbes invariantes par les difféomorphismes de l'anneau. Astérisque, Vol. 103-104 (1983)

6. Lazutkin, V.F.: The existence of caustics for a billiard problem in a convex domain. Izv. Akad. Nauk. SSSR, Ser. Mat. Tom 37 (1973), No. 1 (Russian) [English translation: Math. USSR Izvestija 7, No. 1, 185-214 (1973)]

7. Mather, J.: Glancing billiards. Preprint 1983

8. Mather, J.: Lectures given at ETH Zürich in summer term 1985

9. Mather, J.: Dynamics of area preserving mappings. Preprint 1986

Communicated by J.-P. Eckmann

Received September 2, 1986 